$\checkmark$ Research Square

\title{
Idiopathic Granulomatous Mastitis Presented with Reactive Arthritis and Erythema Nodosum: Case Report from Iraqi
}

\section{Faiq I. Gorial ( $\nabla$ faiqig@gmail.com )}

College of Medicine, University of Baghdad

Farah J.

College of Medicine, Al Mustansiriya University,

\section{Case Report}

Keywords: granulomatous mastitis, erythema nodosum, reactive arthritis, autoimmune mastitis

Posted Date: March 15th, 2021

DOI: https://doi.org/10.21203/rs.3.rs-326299/v1

License: (c) (i) This work is licensed under a Creative Commons Attribution 4.0 International License.

Read Full License 


\section{Abstract}

Idiopathic Granulomatous Mastitis (IGM) is a rare chronic inflammatory disease that involves the development of an inflammatory mass in the breast, which may be difficult to differentiate from malignancy. Few patients have been reported in the literature presenting with arthritis accompanying IGM of breast. Here we report a case of Iraqi patient presented as IGM with reactive arthritis and erythema nodosum.

\section{Introduction}

Idiopathic Granulomatous mastitis is a rare benign chronic inflammatory disease that mainly affects women of childbearing age usually in their early thirties (1), typically it presents as unilateral tender extraareola lump, although bilateral disease has been also described. Regional lymphadenopathy may be present in up to $15 \%$ of cases (2). Selection of a standard method for diagnosing IGM is sophisticated. It's difficult to differentiate from breast cancer as it shares similar clinical and radiologic findings (3) Diagnosis is based mainly on the combination of demonstrating a characteristic histological pattern with exclusion of other possible causes of granulomatous breast lesions $(2,3)$.

\section{Case Report}

A 33-year-old multiparous woman was referred to the Rheumatology unit at Baghdad teaching hospital as a consultation case from breast clinic. The condition started with two- month's history of a palpable painful mass in the left breast that increased in size gradually with no overlying skin changes. She attended the breast clinic for evaluation, breast ultrasound revealed mastitis for which multiple courses of antibiotics has been prescribed without improvement. A week later she developed multiple tender red nodules on the lower extremities with both ankle joints swelling and a low grade fever (Fig. 1), several types of analgesia and low dose prednisolone was started by her physician with no benefit. Past medical history was insignificant. There was no history of recent parturition, breastfeeding, or oral contraceptive pills use in the past three years.

An informed consent was obtained from the patient.

On examining the patient at time of presentation, a tender mass was noted $(3 \times 2 \mathrm{~cm})$ in upper lateral quadrant of the left breast that was firm mobile with smooth surface and normal overlying skin. Two lymph nodes at left axillary region were palpable. Multiple erythematous tender nodules were observed on the extensor surface on both legs consistent with Erythema Nodosum (EN). Patient had obvious arthritis of both ankles. Vital signs examination was normal. Acute phase reactants were elevated [erythrocyte sedimentation rate: $70 \mathrm{~mm} /$ hour (normal, 0-20), C-reactive protein levels: $15 \mathrm{mg} / \mathrm{L}$ (normal, 0-3)]. Leukocyte count was normal as well as the rest of biochemistry blood tests.

Tuberculin skin test (TST) was anergic $(0 \mathrm{~mm})$. IGRA test was also negative. Extra pulmonary tuberculosis of breast was excluded with negative tuberculosis gene expert study of the aspirated fluid 
(repeated twice), and negative acid fast bacilli staining from aspiration material of the lesion. Chest $X$ ray was normal. Angiotensin converting enzyme (ACE) as well as serum calcium level was within the normal range. Sarcoidosis and tuberculosis were excluded with these findings. Bacterial and fungal cultures were negative. Autoimmune markers (antinuclear antibody, extractable nuclear antibodies and anti-neutrophil cytoplasmic antibody) were negative using immunofluorescence method, rheumatoid factor and anticitrullinated antibody level was within normal range. Serum IGG4 level was normal.

Upon repeating Ultrasound examination, mastitis with granuloma was seen in the left breast. Diagnostic fine needle aspiration (FNA) was performed. Cytological examination was nonspecific with granulomatous inflammation consisting of neutrophils, lymphocytes, and multinucleated histiocytic giant cells.

An excisional biopsy was performed with the results demonstrating numerous non necrotizing granulomas with multinucleated giant cells with no significant atypia, malignancy or fibrosis. Through biopsy and exclusion of other possible diseases leading to granulomatous inflammation mentioned above, patient was diagnosed as IGM accompanied by EN and reactive arthritis.

Initial treatment was meloxicam $7.5 \mathrm{mg}$ daily, prednisolone $20 \mathrm{mg}$ daily, azathioprine Table $50 \mathrm{mg}$ twice daily and colchicine Table $0.5 \mathrm{mg}$ per day. On follow-up EN and arthritis regressed completely. Patient was free of symptoms through the duration of follow up.

\section{Discussion}

Idiopathic granulomatous mastitis (IGM) is a subtype of panniculitis with granulomatous inflammation; Kessler and Wolloch first described it as an uncommon disease of females in child bearing age which can mimic various malignant and inflammatory disorders (4). The initial differential diagnosis in more than $50 \%$ of reported cases is malignancy or suspicion of breast carcinoma, with $15 \%$ of patients may present with regional lymphadenopathy (5). IGM is a diagnosis of exclusion requiring careful histopathology review of biopsy specimens and microbiological analysis (6). To exclude other causes of granulomatous inflammation of breast (box 1) (2).In our case, underlying causes were excluded with medical history, laboratory tests, radiology, and pathology. Histopathology is the corner stone in the diagnosis as FNA won't differentiate it confidently from malignancy (7).Because IGM mimics bacterial mastitis, commonly seen in young breastfeeding patients, early treatment with antibiotics is often employed, without success, leading to further workup $(6,8)$.

Box $1^{(2)}$ : Etiologic differential diagnosis of granulomatous lesion of the breast. 


\section{Infectious}

\section{Mycobacterium tuberculosis}

Blastomycosis

Cryptococcosis

Histoplasmosis

Actinomycosis

Filarial infection

Corynebacterium

\section{Autoimmune process}

Wegener granulomatosis

Giant cell arteritis

Foreign body reaction

\section{Duct ectasis}

Plasma cell mastitis

Subareolar granuloma

Periductal mastitis

Diabetes mellitus

Sarcoidosis

Fat necrosis

Idiopathic

Coincidence of granulomatous mastitis, EN and arthritis is a rare feature $(4,9)$. The exact pathogenesis of IGM is unknown, although it is thought to be immunologically mediated. This association of IGM with EN supports the theory that there is an autoimmune component in the pathogenesis of IGM (10). Considering the literature reports, erythema nodosum occurred relatively early in the disease and associated with more rapid progression and a greater degree of inflammation of IGM (8).

A chest x-ray should be performed in all patients to rule out pulmonary diseases as the cause of the cutaneous and breast diseases. Tuberculosis is an important cause of EN and granulomatous mastitis, especially in endemic regions as in our country. In case of our patient; no organisms were seen on Gram or Ziehl-Neelsen stainings. Bacterial, fungal and mycobacterium cultures of the aspiration specimen were negative. Chest $x$-ray was normal and IGRA test was negative, so TB was excluded. There is no definite accepted treatment for IGM (9).It has been proposed that steroid is an effective treatment for IGM. In the current case, treatment with corticosteroid produced a rapid resolution of the fever, EN, breast symptoms and arthritis. 
In conclusion, IGM may rarely associate with EN. Tissue biopsy or cytological examination remains the gold standard for diagnosis. We advocate for an initial trial of immunosuppressive therapy, as it was very successful in our patient.

\section{Declarations}

\section{Conflict of interests}

The authors declare no potential conflict of interests

\section{References}

1. G. Verfaillie, C. Breucq, R. Sacre, et al. Granulomatous Lobular Mastitis: a Rare Chronic Inflammatory Disease of the Breast which Can Mimic Breast Carcinoma, Acta Chirurgica Belgica 2006, 106:2, 222224, DOI: 10.1080/00015458.2006.11679876

2. Bakaris $S$, Yuksel M, Ciragil P,et al.Granulomatous mastitis including breast tuberculosis and idiopathic lobular granulomatous mastitis. Can J Surg. 2006;49(6):427-430.

3. Hovanessian Larsen LJ, Peyvandi B, Klipfel N, et al. Granulomatous lobular mastitis: imaging, diagnosis, and treatment. AJR Am J Roentgenol. 2009; 193(2):574-581. doi:10.2214/AJR.08.1528.

4. Vural S, Ertop P, Ceyhan K, Şanli H. An Unusual Cause of Oligoarthritis and Erythema Nodosum: Idiopathic Granulomatous Mastitis. Arch Rheumatol. 2017; 32(1):71-75. Published 2017 Jan 13. doi:10.5606/ArchRheumatol.2017.5952

5. Chalmers R, McClellan P, Silva V, et al. Red flags for the differential diagnosis of granulomatous mastitis: a case report. J of Med Case Reports. 2020 Nov 10; 14.

6. Fazzio RT, Shah SS, Sandhu NP, Glazebrook KN. Idiopathic granulomatous mastitis: imaging update and review. Insights Imaging. 2016 Aug; 7(4):531-9.

7. Mahmodlou R, Dadkhah N, Abbasi F, et al. Idiopathic granulomatous mastitis: dilemmas in diagnosis and treatment. Electron Physician. 2017;9(9):5375-5379. Published 2017 Sep 25. doi:10.19082/5375.

8. Noma M, Masahiro O, Matsuura K, Itamoto T. Granulomatous Mastitis with Erythema Nodosum That Responded to Low-Dose Steroid: Case Report and Literature Review of Nine Patients. Case Reports in Clin Med. 2014 Jan 1;03:402-6.

9. Binesh F, Shiryazdi M, Bagher Owlia M, Azimi S. Idiopathic granulomatous mastitis, erythema nodosum and bilateral ankle arthritis in an Iranian woman. BMJ Case Rep. 2013;2013:bcr2012007636. Published 2013 Jan 25. doi:10.1136/bcr-2012-007636

10. Zabetian S, Friedman BJ, McHargue C. A case of idiopathic granulomatous mastitis associated with erythema nodosum, arthritis, and reactive cough. JAAD Case Rep. 2016;2(2):125-127. Published 2016 Mar 5. doi:10.1016/j.jdcr.2016.01.011 


\section{Figures}

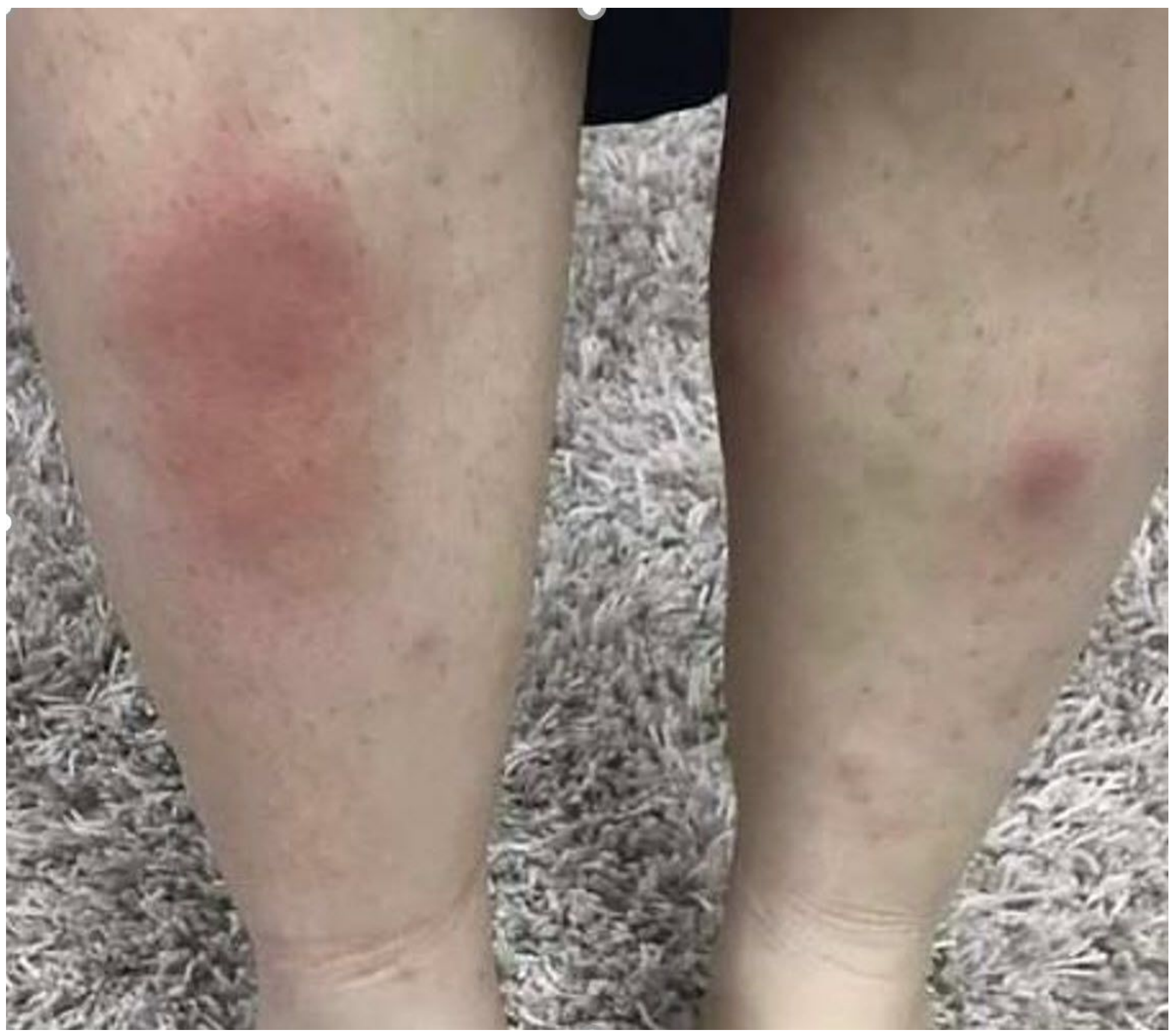

\section{Figure 1}

Erythema nodosum on the extensor surface of both legs 